Subscriber access provided by King Abdullah University of Science and Technology Library

Article

Micro-pseudocapacitors with Electroactive Polymer
Electrodes: Towards Ac-Line Filtering Applications

Narendra Kurra, Qiu Jiang, Ahad Syed, Chuan Xia, and Husam N. Alshareef

ACS Appl. Mater. Interfaces, Just Accepted Manuscript • DOI: 10.1021/acsami.5b12784 • Publication Date (Web): 05 May 2016

Downloaded from http://pubs.acs.org on May 10, 2016

\title{
Just Accepted
}

"Just Accepted" manuscripts have been peer-reviewed and accepted for publication. They are posted online prior to technical editing, formatting for publication and author proofing. The American Chemical Society provides "Just Accepted" as a free service to the research community to expedite the dissemination of scientific material as soon as possible after acceptance. "Just Accepted" manuscripts appear in full in PDF format accompanied by an HTML abstract. "Just Accepted" manuscripts have been fully peer reviewed, but should not be considered the official version of record. They are accessible to all readers and citable by the Digital Object Identifier (DOI®). "Just Accepted" is an optional service offered to authors. Therefore, the "Just Accepted" Web site may not include all articles that will be published in the journal. After a manuscript is technically edited and formatted, it will be removed from the "Just Accepted" Web site and published as an ASAP article. Note that technical editing may introduce minor changes to the manuscript text and/or graphics which could affect content, and all legal disclaimers and ethical guidelines that apply to the journal pertain. ACS cannot be held responsible for errors or consequences arising from the use of information contained in these "Just Accepted" manuscripts. 


\title{
Micro-pseudocapacitors with Electroactive Polymer Electrodes: Towards Ac-Line Filtering Applications
}

\author{
Narendra Kurra ${ }^{+}$, Qiu Jiang ${ }^{+}$, Ahad Syed, Chuan Xia and Husam N Alshareef* \\ Materials Science and Engineering, King Abdullah University of Science and Technology \\ (KAUST), Thuwal 23955-6900 \\ ${ }^{+}$contributed equally to the work \\ E-mail: husam.alshareef@kaust.edu.sa
}

KEYWORDS: ac-line filtering, conducting polymer, electrochemical capacitor, thin film, micro-pseudocapacitor

\begin{abstract}
In this study, we investigate the frequency response of micro-pseudocapacitors (MPCs) based on conducting polymer electrodes such as poly(3,4-ethylenedioxythiophene) (PEDOT), polypyrrole (PPY) and polyaniline (PANI). It is shown that by proper choice of polymeric material and device structure, miniaturized micro-pseudocapacitors can match the frequency response of commercial bulky electrolytic capacitors. Specifically, we show that PEDOT-based micro-pseudocapacitors exhibit phase angle of $-80.5^{\circ}$ at $120 \mathrm{~Hz}$ which is comparable to commercial bulky electrolytic capacitors, but with an order of magnitude higher capacitance density $\left(3 \mathrm{FV} / \mathrm{cm}^{3}\right)$. The trade-off between the areal capacitance $\left(\mathrm{C}_{\mathrm{A}}\right)$ and frequency response in the $2 \mathrm{D}$ architecture $\left(\mathrm{C}_{\mathrm{A}}=0.15 \mathrm{mF} / \mathrm{cm}^{2}\right.$, phase angle of $-80.5^{\circ}$ at $\left.120 \mathrm{~Hz}\right)$ is improved by designing $3 \mathrm{D}$ thin film architecture $\left(\mathrm{C}_{\mathrm{A}}=1.3 \mathrm{mF} / \mathrm{cm}^{2}\right.$, phase angle of $-60^{\circ}$ at $\left.120 \mathrm{~Hz}\right)$. Our work demonstrates that fast frequency response can be achieved using electroactive polymer electrodes.
\end{abstract}




\section{INTRODUCTION}

Aluminium electrolytic capacitors (AECs) are currently being employed in ac-line filtering applications. $^{1-4}$ However, because of their bulky size and limited areal (or volumetric) capacitances, miniaturized electrochemical capacitors are under consideration to replace AECs. ${ }^{5-}$ ${ }^{6}$ Typically, for ac-line filtering, the capacitor must exhibit an impedance phase angle of at least $80^{\circ}$ at a frequency of $120 \mathrm{~Hz}$ (ideal phase angle of $-90^{\circ} @ 120 \mathrm{~Hz}$ ). ${ }^{1-6}$ Traditional supercapacitors, trade name for electrochemical double layer capacitors, exhibit superior areal (or volumetric) capacitance over AECs, but are often characterized by a phase angle of $0^{\circ}$ at 120 Hz. ${ }^{7}$ This is because the internal and intricate porous structure of activated carbon electrodes limits the rate of ion diffusion, making electrochemical double layer capacitors unfit for ac-line filtering. ${ }^{1-2}$ However, other nanocarbon incarnations such as carbon nanotubes, graphene, and their derivatives have been explored for ac-line filtering applications due to their excellent electrical conductivity $(10-300 \mathrm{~S} / \mathrm{cm})$ and fast ion diffusion kinetics. ${ }^{1,5-6}$ Miller et al. have demonstrated vertically grown graphene (thickness, $600 \mathrm{~nm}$ ) by chemical vapour deposition (CVD) on Ni as a suitable device for ac-line filtering. ${ }^{1}$ Similarly, other open porous carbon nanostructures such as ultra-thin films of CNT and reduced graphene oxide have been demonstrated with good frequency response characteristics. ${ }^{8-13}$ Recently, fast response supercapacitors based on graphitic ordered mesoporous carbons with voltage ratings up to $40 \mathrm{~V}$ were demonstrated. ${ }^{12}$ Lim et al., have shown the ac-line filtering capability of crystalline graphene nanostructures carved by unzipping of carbon nanotubes. ${ }^{13}$ Moreover, for compatible integration with other electronic components on a microchip (integrated circuit), electrochemical capacitors must be fabricated in planar format. ${ }^{5}$ Thus, micro-electrochemical capacitor with planar diffusion of electrolyte ions could exhibit high power capabilities over conventional 
sandwich configuration. ${ }^{14}$ Micro-power sources with ac-line filtering capability are highly desirable for developing miniaturized electronic devices. Tour et al., have demonstrated 3D graphene/CNT based micro-electrochemical capacitors for ac-line filtering applications. ${ }^{5}$ The key criterion lies in interplay between the electronic and ionic conductivity of electrochemical capacitor to achieve the desired frequency response. ${ }^{1,5}$

The common feature of all these attempts are based on non-Faradaic carbonaceous materials, both in the thin film and 3D architectures for demonstrating ac-line filtering applications. However, pseudocapacitive materials offer higher capacitance than carbon-based electrodes, and could therefore be a better option if their frequency response can match that of electrolytic capacitors. Indeed, it has been challenging to realize pseudocapacitive electrodes that match the electrolytic capacitors frequency response due to the fact that kinetics of redox events tend to be limited by the rate of ion diffusion across conventional capacitor structure.

Among pseudocapacitive materials, conducting polymers exhibit superior electrical conductivity over transition metal oxides/hydroxides, and are therefore promising materials to be investigated for high power applications. ${ }^{15}$ In addition, conducting polymer electrodes offer additional advantages over carbon-based materials in terms of ease and cost-effective solution processing at room temperature, unlike the high temperature growth processes and complex solution processing methods required for the latter. Thus, in this study, we have systematically investigated the frequency response and pseudocapacitance of micro-pseudocapacitors (MPCs) based on conducting polymer electrodes with $2 \mathrm{D}$ and $3 \mathrm{D}$ current collector designs, including PEDOT, PPY, and PANI. Among the electro-active materials studied, we demonstrate that PEDOT micro-pseudocapacitors in $2 D$ architecture exhibit frequency response matching that of 
commercial electrolytic capacitors, while 3D architecture shows the potential for improving both frequency response and areal capacitance.

\section{EXPERIMENTAL SECTION}

\section{Materials}

Chemicals were used as received without further purification. Analytical grade $\mathrm{H}_{2} \mathrm{SO}_{4}$ and 3,4-ethylenedioxythiophene (EDOT), Aniline (ANI), Pyrrole (PY) and surface active reagent sodium dodecyl sulfate (SDS, $\left.\mathrm{CH}_{3}\left(\mathrm{CH}_{2}\right)_{11} \mathrm{OSO}_{3} \mathrm{Na}\right)$ were purchased from Sigma-Aldrich.

\section{Device fabrication}

\section{2-D Interdigitated Thin Film Electrodes}

Glass substrates $1 \times 1$ 1" size (Fischer), cleaned with a soap solution to remove the dirt followed by ultrasonication in acetone, isopropanol and deionized water sequentially for 5 minutes each and then dried by blowing nitrogen. Photoresist ECI AZ3027 was spun coated at $3000 \mathrm{rpm}$ for 30 seconds over the glass substrate to get $4 \mu \mathrm{m}$ thick photoresist layer. Photoresist coated substrates were soft baked at $100{ }^{\circ} \mathrm{C}$ for 1 minute. The exposure was done using EVG contact aligner at a constant dose of $200 \mathrm{~mJ} / \mathrm{cm}^{2}$ through the $\mathrm{Cr} /$ glass mask having the patterns of interest. After the exposure, samples were developed in AZ726 developer solution for 1 minute, which has resulted in the formation of patterns on the photoresist layer. Metal layers of $250 \mathrm{~nm}$ $\mathrm{Au} / 20 \mathrm{~nm}$ Ti were deposited by sputtering (Equipment Support Co., Cambridge, England) technique over the patterned photoresist layer. Lift-off was done using acetone followed by washing with DI water and dried by blowing $\mathrm{N}_{2}$ gas. Geometric parameters of interdigitated 
fingers have a typical width of $100 \mu \mathrm{m}$ and length of $5000 \mu \mathrm{m}$, while varied interspacings of 10$100 \mu \mathrm{m}$.

\section{3-D Interdigitated Silicon Electrodes}

Si (100) (p-type, resistivity $=5 \mathrm{~m} \Omega . \mathrm{cm}$ ) wafer (thickness, $525 \mu \mathrm{m}$ ) was etch-through employing photolithography and deep reactive ion etching (DRIE). DRIE is an effective process in fabricating 3D structures. ${ }^{14,15}$ Intially, a Si substrate was coated with $10 \mu \mathrm{m}$ thick photoresist (AZ9260) at $2400 \mathrm{rpm}$ for $60 \mathrm{~s}$, followed by $180 \mathrm{~s}$ soft baking at $110^{\circ} \mathrm{C}$. Then a Si wafer which needs to be etched through was bonded onto the photoresist-coated Si substrate. Further, the top Si wafer was coated again with the same photoresist followed by exposure through the etch mask at a constant dose of $1800 \mathrm{~mJ} / \mathrm{cm}^{2}$. The resist was develpoed using AZ726 developer to remove the exposed regions of the photoresist, and to gain access to the Si regions to be etched away by deep reactive ion etching (DRIE). The entire thickness of Si wafer was "etch-through" to obtain interdigitated 3D Si fingers. ${ }^{16-17}$ DRIE was performed in an Oxford Instruments PlasmaLab100 at a temperature of $-20{ }^{\circ} \mathrm{C}$. Sequential $\mathrm{SF}_{6}$ etching and $\mathrm{C}_{4} \mathrm{~F}_{8}$ passivation steps are done with a time duration of $7 \mathrm{~s}$ and $5 \mathrm{~s}$, respectively. The passivation step deposits a hydrophobic fluoropolymer coating that protects against sidewall erosion as etching goes deeper into the silicon wafer. ${ }^{16}$ The process conditions for the deposition cycle included a gas flow $\left(\mathrm{C}_{4} \mathrm{~F}_{8} / \mathrm{SF}_{6}\right)$ of 100/5 sccm flowing at $30 \mathrm{mTorr}$, plasma power of $5 \mathrm{~W}$, and coil power of $1300 \mathrm{~W}$. Similarly, etch cycle plasma was generated by a power of $30 \mathrm{~W}$ and $\left(\mathrm{C}_{4} \mathrm{~F}_{8} / \mathrm{SF}_{6}\right)$ gas flow of $5 / 100 \mathrm{sccm}$ with the other process conditions similar to deposition cycle. The DRIE was done for a total of 1400 cycles to etch through the silicon and stop at the photoresist used for bonding with the 
bottom Si wafer. Further, the 3D interdigitated Si wall structures were metallised by sputtering $250 \mathrm{~nm} \mathrm{Au} / 20 \mathrm{~nm} \mathrm{Ti}$. Metal layers deposited over the photoresist present in the interspaces was removed by mild washing in acetone. This final step has resulted in the formation of $3 \mathrm{D} \mathrm{Au} / \mathrm{Si}$ interdigitated wall structures.

\section{Electrochemical Deposition of Conducting Polymers}

Anodic polymerization of aqueous electrolytic monomeric baths was employed in electropolymerizing of PEDOT, PPY and PANI films using standard three electrode configuration in a glass cell. ${ }^{18-20}$ Substrates consisting of $\mathrm{Au} /$ patterned photoresist/glass or $\mathrm{Au} / \mathrm{Si}$ were used as working electrode, platinum wire as a counter electrode, and $\mathrm{Ag} / \mathrm{AgCl}$ as a reference electrode at room temperature. PEDOT was electrochemically grown on Au surface employing an equimolar $(10 \mathrm{mM})$ solution of EDOT and SDS (supporting electrolyte, 1M $\mathrm{H}_{2} \mathrm{SO}_{4}$ ). Potentio-dynamic mode was used in which the potential was swept from 0 to $1.1 \mathrm{~V}$ at a scan rate of $100 \mathrm{mV} / \mathrm{s}(\mathrm{vs} . \mathrm{Ag} / \mathrm{AgCl})$ for different number of cycles (CHI $660 \mathrm{D}$ Electrochemical

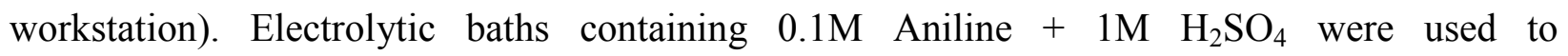
electrodeposit PANI at a scan rate of $100 \mathrm{mV} / \mathrm{s}$ from $-0.2 \mathrm{~V}$ to $0.9 \mathrm{~V}$ versus $\mathrm{Ag} / \mathrm{AgCl}{ }^{21}$ The electrodeposition of PPY was carried out potentiodynamically between the potential limits of 0 and $1 \mathrm{~V}$ vs $\mathrm{Ag} / \mathrm{AgCl}$ at scan rate of $200 \mathrm{mV} / \mathrm{s}$ in an electrolytic bath containing $0.05 \mathrm{M}$ pyrrole + $1 \mathrm{M} \mathrm{H}_{2} \mathrm{SO}_{4}+0.0001 \mathrm{~g} / \mathrm{ml}$ SDS. After the electrochemical deposition, the samples were thoroughly washed with DI water in order to remove any surfactant molecules adsorbed on the surface. Thickness of the deposited polymers was controlled through number of deposition cycles.

\section{Material Characterization}


Surface morphology and microstructure of the polymer films were imaged by scanning electron microscope (SEM) (Nova Nano 630 instrument, FEI Co., The Netherlands). AFM imaging (MFP-3D, Asylum Research) was done using Si probes (model, RTESPA, spring constant 40 $\mathrm{N} / \mathrm{m}$ ) in tapping mode. AFM 3D and roughness analysis was done using WSxM software 4.0 Beta 7.0. ${ }^{22}$ The film thicknesses were measured using a Veeco Dektak 150 surface profilometer. To confirm the electropolymerization of conducting polymers, Raman spectra were recorded using a micro-Raman spectrometer (LabRAM ARAMIS, Horiba-Jobin Yvon). Raman spectra acquired with notch filters cutting at $100 \mathrm{~cm}^{-1}$ using a Cobalt laser (473 nm, $5 \mathrm{~mW}$ at source) and a laser spot size of $1.5 \mu \mathrm{m}$.

\section{Electrochemical Performance of Micro-pseudocapacitor devices}

The electrochemical performance of the conducting polymer micro-pseudocapacitors were investigated in two electrode configuration in $1 \mathrm{M} \mathrm{H}_{2} \mathrm{SO}_{4}$. The gel electrolyte of polyvinyl alcohol (PVA)/ $\mathrm{H}_{2} \mathrm{SO}_{4}$ was prepared as follows: $1 \mathrm{~g}$ of $\mathrm{H}_{2} \mathrm{SO}_{4}$ was added into $10 \mathrm{~mL}$ of deionized water, followed by $1 \mathrm{~g}$ of PVA powder. The whole mixture was heated to $85^{\circ} \mathrm{C}$ while stirring until the solution became clear. As the deposited polymer thickness is very small, gravimetric capacitance is not an appropriate parameter in evaluating the performance of these devices. Hence, we have calculated the areal cell and volumetric capacitances of the micropseudocapacitors by taking the total area and volume of the electroactive electrodes into consideration. Cyclic voltammetry $(\mathrm{CV})$ and galvanostatic charge-discharge (CD) measurements were performed using an electrochemical workstation (model 660D, CH Instruments, Austin, TX, USA). The CVs were tested in a voltage window between 0 and $0.8 \mathrm{~V}$ in a wide range of scan rates. The CDs were measured in the same voltage window under a wide range of current densities. The electrochemical impedance spectroscopy (EIS) data was measured using a 
Modulab (Solartron Analytical) electrochemical workstation in the frequency range from 100 $\mathrm{kHz}$ to $0.01 \mathrm{~Hz}$ at an open circuit potential by applying a small sinusoidal potential of $10 \mathrm{mV}$ signal. All measurements were carried out at room temperature.

\section{RESULTS AND DISCUSSION}
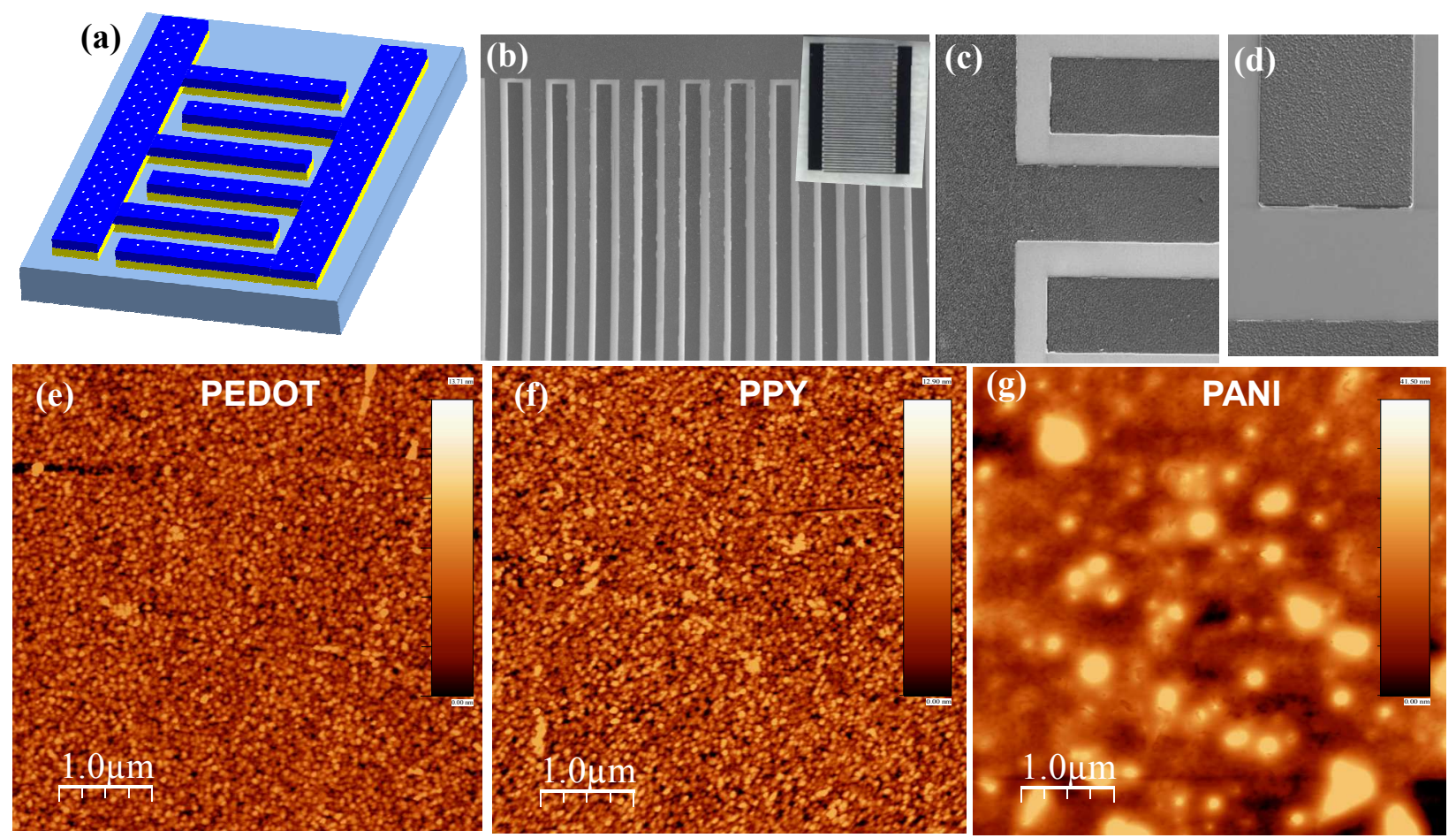

Figure 1. (a) Schematic representation of conducting polymer micro-pseudocapacitor. (b) and (c) SEM micrographs of PEDOT interdigitated fingers with an interspacing of $50 \mu \mathrm{m}$. Digital photograph of PEDOT MSC is shown in the inset. (d) Tilt view of PEDOT thin film deposited over Au finger. (e)-(g) AFM topography images of PEDOT, PPY and PANI thin films electrodeposited on Au surface.

The layout of conducting polymer micro-pseudocapacitor is schematically illustrated in

Figure 1a. Micro-devices with interdigitated electrode structure were fabricated employing 
conventional photolithography (see supporting information, Figure S1). Briefly, a $4 \mu \mathrm{m}$ thick layer of photoresist was spun-coated over the glass substrate followed by UV exposure (dose, $200 \mathrm{~mJ} / \mathrm{cm}^{2}$ ) for 30 seconds. Next, the exposed regions of the photoresist were removed using a developer solution, with subsequent metal deposition (250 nm Au/ $20 \mathrm{~nm} \mathrm{Ti}$ ) by sputtering. At this stage, conducting polymer films (PEDOT, PPY and PANI) were electropolymerised from their respective monomer electrolytic baths. Finally, lift-off using acetone with mild sonication resulted in the neat interdigitated fingers without having any material in the interspace regions as seen from the SEM micrographs in Figures 1b-1c. Digital photograph in the inset shows full view of PEDOT micro-device. Conducting polymer thin films with a typical thickness of $100 \mathrm{~nm}$ were deposited through electrochemical deposition (see Raman data in supporting information, Figure S2). Electrochemical polymerisation is preferred over the solution casting polymerisation as the former offers good interfacial contact between the Au surface and the conducting polymer, avoiding possible interfacial impedance increase across the polymer/Au interface. The strong adherence of PEDOT film onto the $\mathrm{Au}$ surface is clearly evident as it could sustain ultrasonication during the lift-off process. SEM tilt-view of a PEDOT/Au finger is shown in Figure 1d. AFM topography images of conducting polymer films are shown in Figures 1e-1g, which show typical particulate morphology expected for a $100 \mathrm{~nm}$ thick film (see supporting information, Figure S2). 

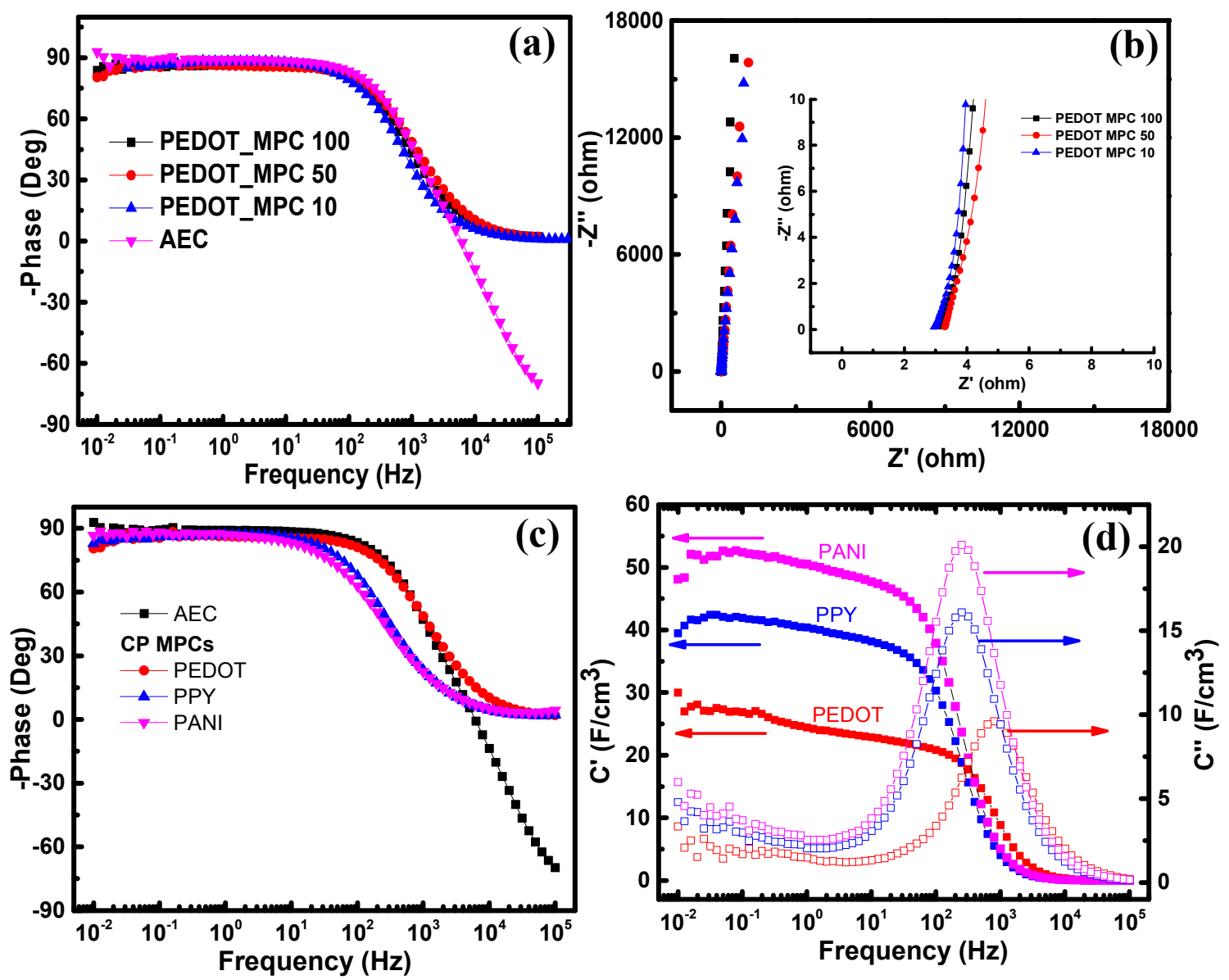

Figure 2. Frequency response characteristics of conducting polymer MPCs. (a) Bode phase angle plots of PEDOT MPCs (100, 50, 10 represent interspacings between the fingers) in comparison with commercial aluminum electrolytic capacitor (AEC) (rated at 2V), (b) Nyquist spectra over the entire frequency range, inset shows the high frequency region of the spectra with nearly vertical nature. Comparison of (c) impedance phase angle and (d) $C^{\prime}$ and $C^{\prime \prime}$ versus frequency of CP MPCs.

The frequency response of conducting polymer MPCs were investigated by electrochemical impedance spectroscopy. The impedance phase angles of PEDOT MPCs (thickness of $\sim 100 \mathrm{~nm}$ ) with interspacings of 100 (MPC 100), 50 (MPC 50) and $10 \mu \mathrm{m}$ (MPC 10) (shown in SEM micrographs in Figure S3) are plotted versus frequency and compared with commercial aluminium electrolytic capacitor (AEC) in Figure 2. Amazingly, the absolute phase angles of these devices are perfectly matching that of commerical AECs as shown in Figure 2a. 
It seems that the spacing between the fingers (in the range of 100-10 $\mu \mathrm{m}$ ) has little influence on the frequency response characteristics of PEDOT MPCs as is evident from Figure 2a. Absolute phase angles of PEDOT MPCs are found to be very close to $-90^{\circ}$ at the low frequency region $(<60 \mathrm{~Hz})$, exhibiting ideal capacitive response. However, for ac-line filtering applications, phase angle should be above $-80^{\circ}$ at $120 \mathrm{~Hz}$. In our case, we have found that PEDOT MSCs exhibited phase angles of $-80.5^{\circ}$ at $120 \mathrm{~Hz}$, comparable to commercial AEC (phase angle of $-84^{\circ}$ at 120 $\mathrm{Hz}$, as shown in Figure 2a). Usually, cross-over frequency at which the impedance phase angle reaches $-45^{\circ}$ is considered for comparison. ${ }^{1,5}$ The typical cross-over frequency (f) of PEDOT MPCs is found to be $1050 \mathrm{~Hz}$. These crossover frequencies are comparable to that of 3D $\mathrm{CNT} /$ graphene $^{5}$ and graphene micro-electrochemical capacitors ${ }^{1}$ reported in the literature. But the performance is far better than some forms of carbonaceous materials such as activated, onion-like carbon, and laser reduced graphene oxide micro-electrochemical capacitors. ${ }^{23-26}$ This frequency response of PEDOT MPCs is comparable to that of ultra-thin films of $\mathrm{CNT}^{9}$ and reduced graphene oxide (rGO) reported in the literature. ${ }^{11}$ The ac frequency response characteristics of PEDOT MPCs are comparable to that of electrical double layer capacitors based on carbonaceous materials with varying thicknesses and configurations (see Tables S1 and S2, supporting information). This behavior indicates that PEDOT MPCs behave more like capacitor with faster ion diffusion kinetics. We believe that the optimal electronic and ionic resistances of PEDOT thin films are responsible for the excellent frequency response characteristics. $^{27}$ Further, we have also investigated the frequency response of PEDOT MPC using solid state electrolyte $\left(\mathrm{PVA} / \mathrm{H}_{2} \mathrm{SO}_{4}\right)$, that exhibit inferior frequency response compared to liquid electrolyte (see Figure S4 in the supporting information). Solid state electrolyte shows lower ionic conductivity over liquid electrolytes due to reduction of ion mobility in the polymer 
matrix. Thus, solid state electrochemical capacitors exhibit lower scan rate capabilities compared to their liquid counterparts. As shown in the Figure S4, solid state device shows constant phase angle of $-80.5^{\circ}$ until $10 \mathrm{~Hz}$ unlike maintained till $120 \mathrm{~Hz}$ in the case of liquid electrolytes. Further, from the Nyquist spectra it is evident that solid state device shows four times higher equivalent series resistance (ESR) compared to the device with liquid electrolyte. At present, commercial electrolytic capacitors also use liquid electrolytes that are being employed in ac-line filtering applications. The challenging aspect lies in developing solid state electrolytes with similar ionic conductivity as that of liquid media that can help in terms of safety and encapsulation while offering the specific capabilities such as ac-line filtering. Further, the stability of PEDOT MPCs was tested by continuously doing charge/discharge between 0 to $0.8 \mathrm{~V}$ at a current density of $0.15 \mathrm{~mA} / \mathrm{cm}^{2}$, as shown in Figure S5. It is clear that PEDOT MPCs show capacitance retention of $90 \%$ and coulombic efficiency of nearly $100 \%$ over 10000 cycles.

Porosity, intrinsic electrical conductivity and redox behavior of the electrode material are the major governing factors that determine the frequency response of an electrochemical capacitor besides the electrode architecture. ${ }^{2,7}$ As shown in Figure 2b, Nyquist plots shown for PEDOT MPCs are seen parallel to $-Z^{\prime \prime}$ axis with the absence of semi-circle in the high frequency region, which implies that excellent ohmic coupling between the Au current collector and PEDOT polymeric layers. Information about the behavior of the electrode materials can be estimated from the line intersecting the real axis at an angle of $45^{\circ}$ in a Nyquist plot. ${ }^{1}$ PEDOT MPC exhibits little or negligible porosity effect as is evident from high frequency region, shown in Figure $2 b$ (also see the inset).

Impedance phase angles of other conducting polymer MPCs such as PPY and PANI were studied and compared with PEDOT, as shown in Figure 2c. It can be seen that PPY and PANI 
MSCs exhibit phase angles of $60-65^{\circ}$ at a frequency of $120 \mathrm{~Hz}$, while the cross-over frequencies are in the range of $250-300 \mathrm{~Hz}$. Certainly, these conducting polymer MSCs (PPY and PANI) exhibit inferior ac-response characteristics compared to PEDOT, which is attributed to the electronic conductivity and intrinsic redox nature of the electrodes. ${ }^{15}$ Typical conductivities of PEDOT, PPY and PANI thin films were found to be 108, 9.2 and $2.9 \mathrm{~S} / \mathrm{cm}$, respectively (see supporting information, Figure S6). ${ }^{15}$ The diminished frequency response of PPY and PANI MPCs can be attributed to the greater redox activity (extent of doping level is more for PANI and PPY compared to PEDOT) and lower conductivity compared to PEDOT. The reason for the absence of a clear semi-circle for PPY and PANI MPCs could be due to the thin film morphology and the in-plane configuration of electrodes while having the ohmic coupling between the current collectors and conducting polymeric layers (see supporting information, Figures S7-S11). Thus, PEDOT MPC behaves more like a capacitor while PPY and PANI MPCs may exhibit charge leakage due to Faradaic charge transfer. ${ }^{2,28}$

The real $\left(\mathrm{C}^{\prime}\right)$ and imaginary $\left(\mathrm{C}^{\prime \prime}\right)$ parts of capacitances are extracted from the electrochemical impedance data of conducting polymer MPCs. Figure $2 \mathrm{~d}$ displays plot of $\mathrm{C}^{\prime}$ and $\mathrm{C}^{\prime \prime}$ versus frequency (calculated using the equations mentioned in the supporting information). Notice that $\mathrm{C}^{\prime}$ of PEDOT MPC remains unchanged up to $120 \mathrm{~Hz}$, suggesting excellent capacitive behavior compared to PPY and PANI MPCs. It is interesting to compare the characteristic relaxation time constant $\left(\tau_{0}=1 / f_{0}\right)$, which is the time required for discharging a capacitor with an efficiency greater than $50 \% .{ }^{16}$ This can be obtained from the frequency $\left(f_{o}\right)$ at which the imaginary capacitance $\mathrm{C}^{\prime \prime}$ becomes maximum. The relaxation time constant is found to be $1 \mathrm{~ms}$ for PEDOT MPCs and 3-4 ms for the PPY and PANI MPCs. However, PPY and PANI MPCs exhibit a volumetric capacitance of $40-50 \mathrm{~F} / \mathrm{cm}^{3}$, superior to PEDOT MPC $\left(30 \mathrm{~F} / \mathrm{cm}^{3}\right)$. PEDOT, 
due to high monomer mass and low doping level, exhibits lower capacity in comparison to PPY and PANI. ${ }^{29}$ Thus, PPY and PANI, due to their intrinsic redox characteristics can contribute to the higher capacity values, while the intrinsic conducting nature of PEDOT is responsible for its excellent ac-response characteristics. ${ }^{15}$ The Resistor-Capacitor $(\mathrm{RC})$ time constant $\left(\tau_{\mathrm{RC}}\right)$ at 120 $\mathrm{Hz}$ is found to be $0.21 \mathrm{~ms}$ for PEDOT MPCs. This value is much shorter than $8.3 \mathrm{~ms}$ period required for $120 \mathrm{~Hz}$ filtering, matching that of commercial AEC (0.15 ms). Carbon based electrochemical capacitors with internal porosity will have distributed charge storage, exhibiting multiple time constants associated with the RC networks corresponding to the dispersed porous structure. ${ }^{1}$ Consequently, quoting of single RC time constant for such a device may not be appropriate; a better concept would be to use "characteristic response time", that is related to the energy extraction rate. Based on this concept, the typical activated carbon type EDLC will have a characteristic response time of $\sim 1$ second. However, the characteristic response time should be $8.3 \mathrm{~ms}$ (to respond capacitively at $120 \mathrm{~Hz}$ ) for ac-line filtering applications. Thus, activated carbon EDLC is incapable of responding at $120 \mathrm{~Hz}$ (it behaves like a resistor rather than a capacitor at $120 \mathrm{~Hz}$ as the characteristic response time of 1 second is far higher than $8.3 \mathrm{~ms}$ ). It is evident from the impedance data shown in Figure 2a; PEDOT MPCs clearly can respond capacitively at $120 \mathrm{~Hz}$ and can be represented by an equivalent series-RC circuit model (with a single time constant of $0.21 \mathrm{~ms})$.

The excellent frequency response of PEDOT MPCs can be attributed to the thin film nature of conducting PEDOT with proper interfacial characteristics as well as the in-plane configuration of electrode architecture; these two factors together contribute to the lower values of electronic and ionic resistances. Recently, there have been siginificant amount of research papers focussing on the frequency response characteristics of open porous carbon forms such as 
graphene and CNTs in various electrode architectures. Pseudocapacitors such as conducting polymers may involve Faradaic charge storage besides the coupling of double layer formation but the electrical response is similar to that of shown by a capacitor. At $120 \mathrm{~Hz}$, PEDOT MPC has a volumetric capacitance of $19 \mathrm{~F} / \mathrm{cm}^{3}$ with an ESR of $5.8 \Omega$ and corresponding time constant of $209 \mu \mathrm{s}$. The capacitance density is found to be $12 \mathrm{FV} / \mathrm{cm}^{3}$ (for active layer thickness only) and $3 \mathrm{FV} / \mathrm{cm}^{3}$ (considering thickness of both current collectors and active material) (aqueous electrolyte, voltage window of $0.8 \mathrm{~V}$ ) which is far superior to the low-voltage (2V, areal capacitance of $40 \mu \mathrm{F} / \mathrm{cm}^{2}$ ) aluminium anode foil (KDK, Tokyo, Japan) with capacitance density values up to $\sim 0.14 \mathrm{FV} / \mathrm{cm}^{3}{ }^{1}$ Typically, AECs rated at $2 \mathrm{~V}$ or higher are being employed in acline filtering of portable electronic devices. Thus, PEDOT MPCs exhibit superior capacitance density by at least an order of magnitude over commercial AECs while matching the frequency response characteristics. 

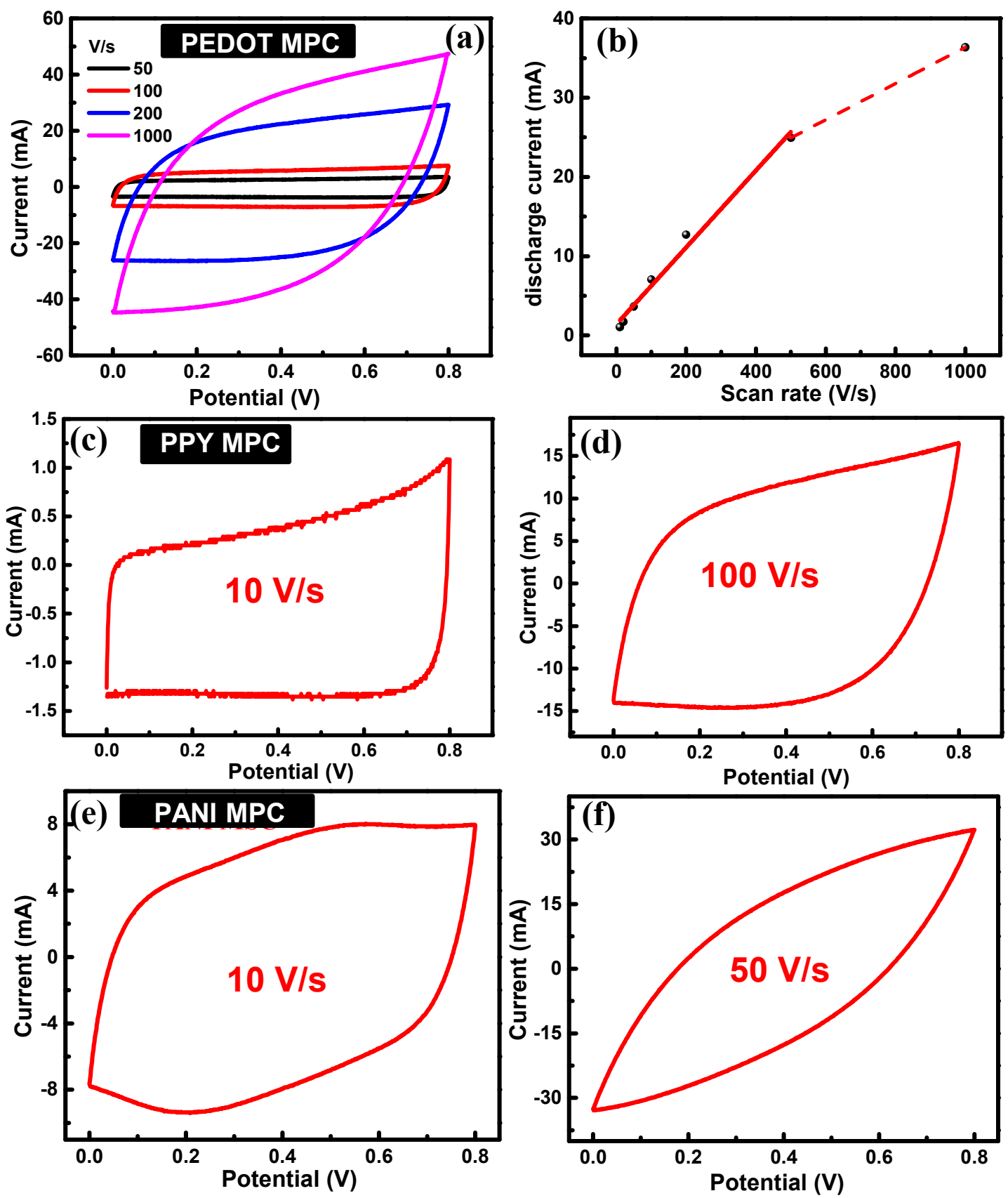

Figure 3. Electrochemical performance of conducting polymer MPCs. (a) CVs and (b) variation of discharge current with scan rate of PEDOT MPC. CVs of PPY (c) and (d) PANI MPCs (e) and (f).

As shown in Figure 3a, CVs of PEDOT MPCs are quite rectangular in nature until ultra high scan rates of $1000 \mathrm{~V} / \mathrm{s}$, indicating the capacitive nature of PEDOT MPCs. Discharge current values are plotted versus scan rate and show linear behavior up to the scan rate of $500 \mathrm{~V} / \mathrm{s}$, 
beyond which a slight deviation from linearity is observed (see Figure 3b). Since the entire volume of the thin film can be accessed by the electrolyte ions without any diffusion limitations, ultra-high scan rate capability is observed for PEDOT MPCs. However, in the case of PPY MPCs, as shown in Figures $3 \mathrm{c}$ and $3 \mathrm{~d}$, rectangle $\mathrm{CV}$ shape is retained only up to $100 \mathrm{~V} / \mathrm{s}$. Similarly, PANI MPC shows broad redox peaks at a scan rate of $10 \mathrm{~V} / \mathrm{s}$, indicating its Faradaic nature of charge storage (see Figure 3e). Further, at a high scan rate of $50 \mathrm{~V} / \mathrm{s}$, PANI MPCs exhibit complete deviation from the rectangular $\mathrm{CV}$ shape, indicating the dominating resistive nature of PANI MPCs at higher scan rates (see Figure 3f). Thus, the intrinsic redox behavior of PANI contributes to the higher values of capacitance at moderate scan rates (up to $10 \mathrm{~V} / \mathrm{s}$ ), but loses its pseudo-capacitive nature at higher scan rates $(>50 \mathrm{~V} / \mathrm{s})$. This result implies that charge leakage by Faradaic charge transfer could limit the kinetics of a pseudocapacitor device. ${ }^{2,30}$
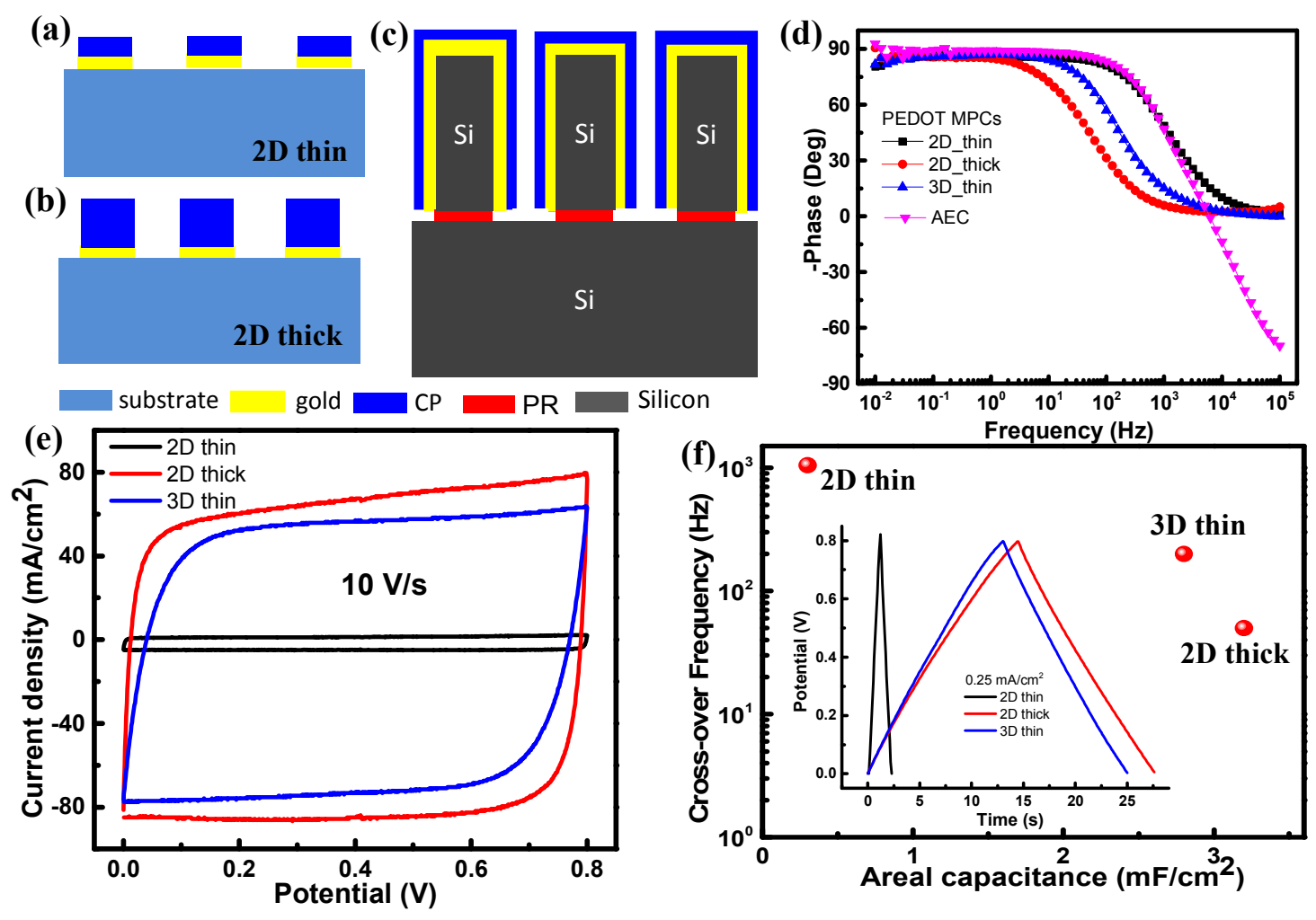

Figure 4. Schematic of PEDOT MPCs with (a) 2D thin, (b) 2D thick and (c) 3D thin film electrodes. (d) Comparison of Bode phase plots of PEDOT MPCs in 2D and 3D architectures. 
(e) CVs of PEDOT MPCs in 2D and 3D formats. (f) Cross-over frequency (corresponding phase angle of $-45^{\circ}$ ) versus areal capacitance of various PEDOT MPCs, inset shows the comparison of CDs of 2D thin and thick, 3D thin PEDOT MPCs.

Despite the fact that PEDOT MPCs with thin polymer films (see schematic in Figure 4a) exhibit good ac response, they have limited areal capacitance $\left(0.3 \mathrm{mF} / \mathrm{cm}^{2}\right.$ for $100 \mathrm{~nm}$ thin film $)$. To increase the capacitance, we attempted two different approaches. In one case, as shown in the schematic of Figure 4b, thicker PEDOT films (thickness, $1 \mu \mathrm{m}$ ) were deposited by increasing electrodeposition time. In another case, 3D interdigitated Si fingers (thickness $>300 \mu \mathrm{m}$ ) were fabricated by employing microelectromechanical (MEM) systems technologies to increase loading of active materials in a given foot-print area. ${ }^{16-17}$ PEDOT thin films were deposited on metallized 3D Si fingers (as described in the experimental section, see Figure S12, supporting information) and shown in Figure 4c. Bode phase angle plots of PEDOT MPCs in these various electrode architectures are compared with the commercial elctrolytic capacitor (AEC) as shown in Figure 4d. As was discussed earlier, 2D thin film PEDOT MPCs exhibit good frequency response characteristics and indeed match the electrochemical performance of commercial AEC. Thicker PEDOT MPCs (thickness, $1 \mu \mathrm{m}$ ) in the $2 \mathrm{D}$ design exhibit a phase angle of $-35^{\circ}$ at 120 $\mathrm{Hz}$ with a cross-over frequency (corresponding phase angle of $-45^{\circ}$ ) of $50 \mathrm{~Hz}$ (see red curve in Figure 4d). Similarly, as shown in Figure S10a, the cross-over frequency of PPY MPCs at $-45^{\circ}$ diminished from 425 to $37 \mathrm{~Hz}$ when the thickness was increased from 100 and 500. Similarly, for PANI MPCs (shown in Figure S11a), cross-over frequency at $-45^{\circ}$ was observed to be 265 $\mathrm{Hz}, 32 \mathrm{~Hz}$ and $2.3 \mathrm{~Hz}$ for $100 \mathrm{~nm}, 300 \mathrm{~nm}$ and $500 \mathrm{~nm}$ films, respectively. The inferior frequency response characteristics of 2D thick PEDOT MPCs can be attributed to the longer ion diffusion lengths and increased electrical resistivity of the thicker films. Similar observations were made in the case of $\mathrm{RuO}_{2}$ pseudocapacitors. ${ }^{2}$ 
Interestingly, the 3D PEDOT MPCs exhibit a phase angle of $-60^{\circ}$ at $120 \mathrm{~Hz}$ with a cross-over frequency of $200 \mathrm{~Hz}$, much better than 2D thick PEDOT MPCs (see blue curve in Figure 4d). It was observed that 2D thick and 3D thin PEDOT MPCs exhibit an enhanced areal capacitance up to 10 times higher than 2D thin film PEDOT MPCs. This is due to maximum loading of electroactive materials in a given foot-print area. As shown in Figure 4e, CVs of PEDOT MPCs are seen to be quite rectangular, behaving like a capacitor irrespective of the design. Areal capacitance of both 2D thick and 3D thin PEDOT MPCs are found to be around $3 \mathrm{mF} / \mathrm{cm}^{2}(0.3$ $\mathrm{mF} / \mathrm{cm}^{2}$ for $2 \mathrm{D}$ thin film) at a current density of $0.25 \mathrm{~mA} / \mathrm{cm}^{2}$ (see the inset of Figure $4 \mathrm{f}$ ). As shown in Figure 4f, 2D thin film PEDOT MPCs exhibit excellent frequency response but suffer from lower areal capacitance while 2D thick PEDOT MPCs exhibit poor frequency response with higher areal capacitance. Thus, the trade-off between the capacitance and frequency response can be optimized by considering the 3D architecture. It is clear that the 3D PEDOT MPCs exhibit similar areal capacitance with superior frequency response compared to 2D thick PEDOT MPCs (see Figure 4e). In other words, 3D PEDOT architecture seems to maintain similar areal capacitance to 2D thick PEDOT MSCs, but with better frequency response. Thus, our results clearly indicate that 3D architecture is a good choice for fabricating microelectrochemical capacitors which can maintain excellent areal capacitance and frequency response.

\section{CONCLUSIONS}

In summary, we have studied the frequency response of micro-pseudocapacitors based on conducting polymer electrodes such as poly(3,4-ethylenedioxythiophene) (PEDOT), polypyrrole (PPY) and polyaniline (PANI), fabricated in both two dimensional (2D) and three dimensional (3D) architectures. Among the polymeric materials studied, PEDOT micro-pseudocapacitors 
exhibit phase angle of $-80.5^{\circ}$ at $120 \mathrm{~Hz}$, comparable to that of commercial bulky electrolytic capacitors, but with an order of magnitude higher capacitance density. This can be attributed to the higher electrical conductivity and fast surface redox reactions of PEDOT over other conducting polymers. Moreover, the existence of trade-off between areal capacitance $\left(\mathrm{C}_{\mathrm{A}}\right)$ and frequency response in the $2 \mathrm{D}$ configuration was optimised to some extent by choosing $3 \mathrm{D}$ architecture. Our work clearly demonstrated that properly designed micro-pseudocapacitors can exhibit frequency response matching that of commercial electrolytic capacitors. Further, this study may open up new avenues in exploring the 3D micro-electrochemical capacitors composed of pseudocapacitive materials to maintain the optimal balance between areal capacitance and frequency response.

\section{Supporting Information}

Figure S1. Schematic illustration of fabrication of conducting polymer micro-pseudocapacitors (MPCs).

Figure S2. SEM micrographs of electrodeposited (a) PEDOT, (b) PPY and (c) PANI thin films. (d) Raman spectra of electrodeposited CPs.

Figure S3. SEM micrographs of PEDOT MPCs with interspacing of (a) 10, (b) 50 and (c) 100 $\mu \mathrm{m}$, finger width of $100 \mu \mathrm{m}$ is same throughout.

Figure S4. Comparison of (a) Bode phase angle vs. frequency and (b) Nyquist spectra of PEDOT MPCs with aqueous (black curve) and solid state (red curve) electrolytes.

Figure S5. Cycling stability and coulombic efficiency of PEDOT MPC over 10000 cycles at a current density of $0.15 \mathrm{~mA} / \mathrm{cm}^{2}$ in $1 \mathrm{M} \mathrm{H}_{2} \mathrm{SO}_{4}$, inset shows the frequency response before and after cycling.

Figure S6. Four probe current-voltage characteristics of conducting polymer thin films. Typical conductivities of PEDOT, PPY and PANI thin films (thickness of $100 \mathrm{~nm}$ ) are found to be 108, 9.2 and $2.9 \mathrm{~S} / \mathrm{cm}$, respectively.

Figure S7. (a) Nyquist spectra of PEDOT, PPY and PANI MPCs, inset shows the high frequency region of the spectra. (b) Comparison of Nyquist spectra of conducting polymer micro-electrochemical capacitors with AEC. 
Figure S8. (a) Equivalent circuit model for fitting the impedance data of conducting polymer microsupercapacitors. Nyquist plots and fitted plot for PEDOT (b), PPY (c), PANI (d).

Figure S9. Percentage contribution of double layer and pseudocapacitive contributions for the conducting polymer microsupercapacitors, obtained after fitting the impedance data to an equivalent circuit shown in Figure S8.

Figure S10. (a) Bode phase angle plots and (b) CVs of PPY MPCs with different thicknesses.

Figure S11. (a) Bode phase angle plots and (b) CVs of PANI MPCs with different thicknesses.

Figure S12. Schematic illustration of Si etch-through process employing photolithography and DRIE. Inset shows micrograph of interdigitated 3D Si wall structures.

Table S1. Electrochemical performance comparison of carbonaceous materials comparison of the electrochemical performance of the micro-electrochemical capacitors reported in the literature.

Table S2. Electrochemical performance of conducting polymer micro-pseudocapacitors in this work.

This material is available free of charge via the Internet at http://pubs.acs.org

\section{ACKNOWELDGEMENTS}

Research reported in this publication was supported by King Abdullah University of Science and Technology (KAUST). Authors thank the Advanced Nanofabrication, Imaging and Characterization Laboratory at KAUST for their excellent support. 


\section{REFERENCES}

1. Miller, J. R.; Outlaw, R.; Holloway, B. Graphene Double-Layer Capacitor with AC LineFiltering Performance. Science 2010, 329, 1637-1639.

2. Conway, B. E. Electrochemical Supercapacitors: Scientific Fundamentals and Technological Applications; Plenum press, Springer: New York, 1999.

3. Miller, J. R.; Burke, A. F. Electrochemical Capacitors: Challenges and Opportunities for Real-World Applications. Electrochem. Soc. Interface. 2008, 17, 53-57.

4. De Levie, R.; Delahay, P. Electrochemical Response of Porous and Rough Electrodes. Advances in electrochemistry and electrochemical engineering 1967, 6, 329-397.

5. $\quad$ Lin, J.; Zhang, C.; Yan, Z.; Zhu, Y.; Peng, Z.; Hauge, R. H.; Natelson, D.; Tour, J. M. 3Dimensional Graphene Carbon Nanotube Carpet-based Microsupercapacitors with High Electrochemical Performance. Nano Lett. 2012, 13, 72-78.

6. Sheng, K.; Sun, Y.; Li, C.; Yuan, W.; Shi, G. Ultrahigh-Rate Supercapacitors based on Eletrochemically Reduced Graphene Oxide for Ac Line-Filtering. Sci. Rep. 2012, 2, 247-251.

7. Boos, D.; Argade, S. In Historical Background and New Perspectives for Double-Layer Capacitors, $1^{\text {st }}$ International Seminar on Double Layer Capacitors and Similar Energy Storage Devices, Deerfield Beach, FL, 1991.

8. Rangom, Y.; Tang, X.; Nazar, L. F. Carbon Nanotube-based Supercapacitors with Excellent AC Line Filtering and Rate Capability via Improved Interfacial Impedance. ACS Nano 2015, 9, 7248-7255.

9. Yoo, Y.; Kim, S.; Kim, B.; Kim, W. 2.5 V Compact Supercapacitors based on Ultrathin Carbon Nanotube Films for AC Line Filtering. J. Mater. Chem. A 2015, 3, 11801-11806.

10. Laszczyk, K. U.; Kobashi, K.; Sakurai, S.; Sekiguchi, A.; Futaba, D. N.; Yamada, T.; Hata, K. Microsupercapacitors: Lithographically Integrated Microsupercapacitors for Compact, High Performance, and Designable Energy Circuits Adv. Energy Mater. 2015, 5, 1500741.

11. Wu, Z. S.; Liu, Z.; Parvez, K.; Feng, X.; Müllen, K. Ultrathin Printable Graphene Supercapacitors with AC Line-Filtering Performance. Adv. Mater. 2015, 27, 3669-3675.

12. Yoo, Y.; Kim, M.-S.; Kim, J.-K.; Kim, Y. S.; Kim, W. Fast-Response Supercapacitors with Graphitic Ordered Mesoporous Carbons and Carbon Nanotubes for AC Line Filtering. $J$. Mater. Chem. A 2016, 4, 5062-5068.

13. Lim, J.; Maiti, U. N.; Kim, N. Y.; Narayan, R.; Lee, W. J.; Choi, D. S.; Oh, Y.; Lee, J. M.; Lee, G. Y.; Kang, S. H.; Kim, H.; Kim, Y. H.; Kim, S. O. Dopant-Specific Unzipping of Carbon Nanotubes for Intact Crystalline Graphene Nanostructures. Nat. Commun. 2016, 7 , 10364.

14. Beidaghi, M.; Gogotsi, Y. Capacitive Energy Storage in Micro-Scale Devices: Recent Advances in Design and Fabrication of Micro-Supercapacitors. Energy Environ. Sci. 2014, 7, 867-884.

15. Snook, G. A.; Kao, P.; Best, A. S. Conducting Polymer based Supercapacitor Devices and Electrodes. J. Power Sources 2011, 196, 1-12.

16. Sun, W.; Zheng, R.; Chen, X. Symmetric Redox Supercapacitor Based on MicroFabrication with Three-Dimensional Polypyrrole Electrodes. J. Power Sources 2010, 195, 71207125 .

17. Shen, C.; Wang, X.; Li, S.; Zhang, W.; Kang, F. A High Energy Density Micro Supercapacitor of Asymmetric $\mathrm{MnO}_{2}$ Carbon Configuration by using Micro Fabrication Technologies. J. Power Sources 2013, 234, 302-309. 
18. Wang, K.; Wu, H.; Meng, Y.; Wei, Z. Conducting Polymer Nanowire Arrays for High Performance Supercapacitors. Small 2014, 10, 14-31.

19. Wang, K.; Zou, W.; Quan, B.; Yu, A.; Wu, H.; Jiang, P.; Wei, Z. An All-Solid-State Flexible Micro-supercapacitor on a Chip. Adv. Energy Mater. 2011, 1, 1068-1072.

20. Patra, S.; Munichandraiah, N. Supercapacitor Studies of Electrochemically Deposited PEDOT on Stainless Steel Substrate. J. Appl. Polym. Sci. 2007, 106, 1160-1171.

21. Kurra, N.; Jiang, Q.; Alshareef, H. N. A General Strategy for the Fabrication of High Performance Microsupercapacitors. Nano Energy 2015, 16, 1-9.

22. Horcas, I.; Fernández, R.; Gomez-Rodriguez, J.; Colchero, J.; Gómez-Herrero, J.; Baro, A., WSXM: A Software for Scanning Probe Microscopy and A Tool for Nanotechnology. Rev. Sci. Instrum. 2007, 78, 013705.

23. Pech, D.; Brunet, M.; Taberna, P.-L.; Simon, P.; Fabre, N.; Mesnilgrente, F.; Conédéra, V.; Durou, H. Elaboration of a Microstructured Inkjet-Printed Carbon Electrochemical Capacitor. J. Power Sources 2010, 195, 1266-1269.

24. Pech, D.; Brunet, M.; Durou, H.; Huang, P.; Mochalin, V.; Gogotsi, Y.; Taberna, P.-L.; Simon, P. Ultrahigh Power Micrometre Sized Supercapacitors Based on Onion-Like Carbon. Nat. Nanotechnol. 2010, 5, 651-654.

25. Gao, W.; Singh, N.; Song, L.; Liu, Z.; Reddy, A. L. M.; Ci, L.; Vajtai, R.; Zhang, Q.; Wei, B.; Ajayan, P. M. Direct Laser Writing of Micro-Supercapacitors on Hydrated Graphite Oxide Films. Nat. Nanotechnol. 2011, 6, 496-500.

26. El-Kady, M. F.; Kaner, R. B., Scalable Fabrication of High-Power Graphene MicroSupercapacitors for Flexible and On-Chip Energy Storage. Nat. Commun. 2013, 4, 1475.

27. Kurra, N.; Hota, M.; Alshareef, H. N. Conducting Polymer Micro-Supercapacitors for Flexible Rnergy Storage and AC Line-Filtering. Nano Energy 2015, 13, 500-508.

28. Taberna, P.; Simon, P.; Fauvarque, J.-F., Electrochemical Characteristics and Impedance Spectroscopy Studies of Carbon-Carbon Supercapacitors. J. Electrochem. Soc. 2003, 150, A292A300.

29. Snook, G. A.; Chen, G. Z., The Measurement of Specific Capacitances of Conducting Polymers using the Quartz Crystal Microbalance. J. Electroanal. Chem. 2008, 612, 140-146.

30. Talbi, H.; Just, P.-E.; Dao, L., Electropolymerization of Aniline on Carbonized Polyacrylonitrile Aerogel Electrodes: Applications for Supercapacitors. J. Appl. Electrochem. 2003, 33, 465-473. 
Table of Content (ToC)
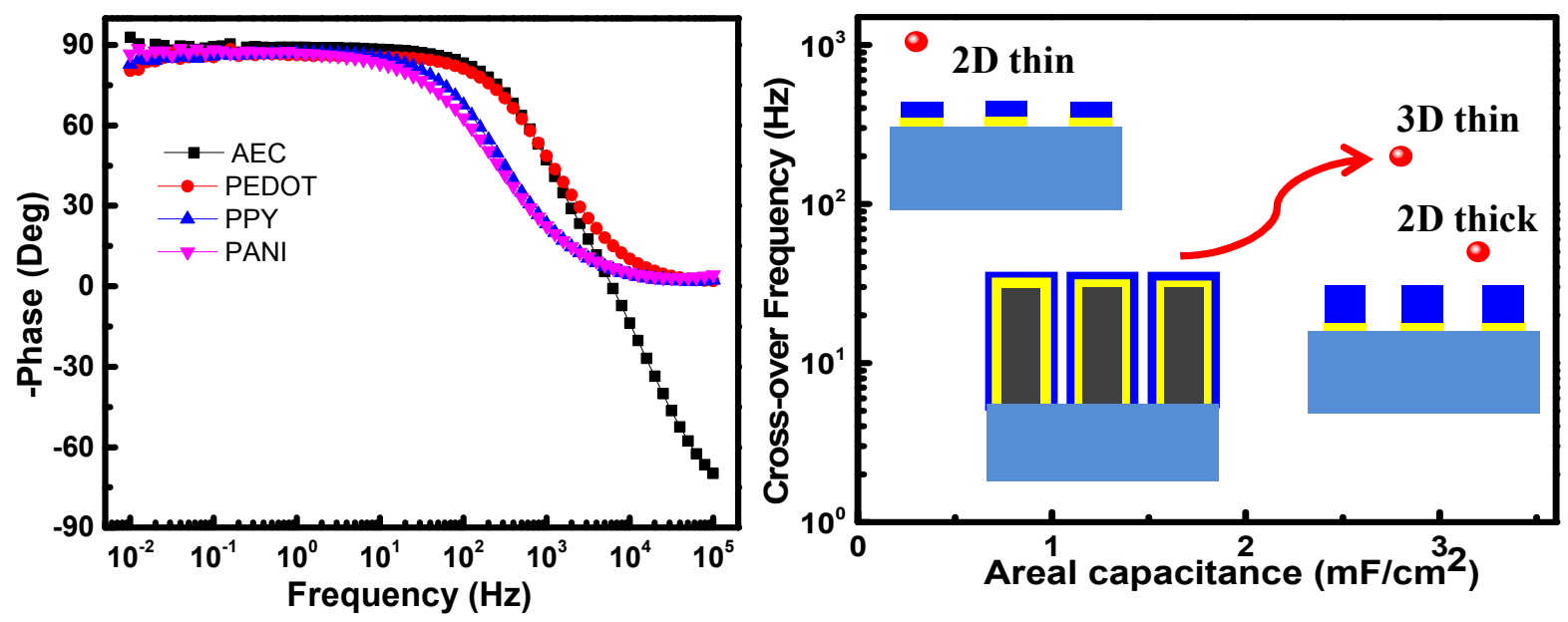